\title{
Mental Health Problems among Adolescents in Relationship to Satisfaction with Parental Control
}

\author{
Vinita Tiwari $^{1 *}$, Saroj Verma ${ }^{2}$
}

\section{ABSTRACT}

Several studies indicated a relation between parental control and symptoms of anxiety and depression among adolescents. But satisfaction with parental control has not been given much emphasis with regard to mental health problems among adolescents. The present study aimed to examine the adolescents' satisfaction with parental control and its relationship with anxiety and depression. It was hypothesized that satisfaction with parental control (maternal and paternal) would be negatively associated with anxiety and depression among adolescents. Four hundred adolescents (200 boys and 200 girls) with age range of 15 to 19 years, were administered 'Satisfaction with Parental Control Scale' and 'General Health Questionnaire' to assess the variables under study. Correlational analysis revealed that adolescents who have greater satisfaction with their maternal and paternal control reported less anxiety and depressive symptoms. Further, regression analysis clearly indicates that satisfaction with maternal control emerged as significant predictor for anxiety and depression. Present study provides the conclusion that satisfaction with parental control improves mental health status of adolescents. The findings may have counselling implications.

Keywords: Satisfaction with paternal control, Satisfaction with maternal control, Anxiety, Depression, Mental health

Adolescence is a time of great change and transition, when youth are starting to make decisions about career paths, further schooling, and living on their own. These stressors, coupled with changing peer and family interactions, may lead in some cases to mental health problems, such as depression, suicidal thoughts, and anxiety disorders etc. Pillai, Patel, Cardozo, Goodman, Weiss and Andrew (2008) reported that anxiety, depression and behavioural disorders and attention-deficit hyperactivity disorder were the major mental health problems that occurred frequently during adolescence. Srinath, Girimaji, Gururaj, Seshadri, Subbakrishna, Bhola and

\footnotetext{
${ }^{1}$ Ph.D., Department of Psychology, Banaras Hindu University, Varanasi, U.P., India

${ }^{2}$ Ph.D., Professor, Department of Psychology, Banaras Hindu University, Varanasi, U.P, India

*Responding Author
}

Received: January 4, 2017; Revision Received: February 4, 2017; Accepted: February 7, 2017

(C) 2017 Tiwari V, Verma S; licensee IJIP. This is an Open Access Research distributed under the terms of the Creative Commons Attribution License (www.creativecommons.org/licenses/by/2.0), which permits unrestricted use, distribution, and reproduction in any Medium, provided the original work is properly cited. 
Kumar (2005) reported that $12.5 \%$ of children and adolescents suffering from mental and behavioural health problems in India.

Anxiety is one of the most common mental-health problems experienced by adolescents. Anxiety is associated with substantial negative effects on children's social, emotional and academic success (Essau, Conradt \& Petermann, 2000). McLeod, Wood and Weisz (2007), revealed that higher levels of parental control were associated with more anxiety in children, Some theoretical models (Chorpita \& Barlow, 1998; Rapee, 2001) hypothesize that when parents are highly controlling, children may experience decreased self-efficacy, and thus, increased anxiety (Wood, 2006). Conversely, some models (e.g., Chorpita \& Barlow, 1998; Wood, McLeod, Sigman, Hwang, \& Chu, 2003) have hypothesized that parental encouragement of children's autonomy and independence (e.g., in novel contexts) may increase children's perceptions of mastery over the environment, leading to anxiety reduction.

Depression in childhood and adolescence is a significant, persistent, and unbearable problem, undermining social and school functioning, and prompting substantial mental health service use (Clarke, DeBar, \& Lewinsohn, 2003). Depression in children is a serious, pervasive problem with an unfortunate developmental course. The evidence for concurrent associations between parenting and adolescent depressive symptoms is devastating (Heaven, Newbury \& Mak, 2004; Pavlidis \& McCauley, 2001). According to attachment theory (Bowlby, 1973), theories regarding the connectedness-individuation link (Grotevant \& Cooper, 1986), and theories dealing with parenting practices (Baumrind \& Black, 1967), positive developmental outcomes with lower levels of depressive symptoms occur when adolescents have close relationships with their parents. Lower quality relationships with parents may lead to more distress because adolescents in such relationships experience low support when facing emotional problems (Flouri \& Buchanan, 2003). The perception of being accepted and valued is thought to increase self-esteem and self-efficacy, which protects against depressive feelings (Wilkinson, 2004).

Adolescents' mental health and well-being are found to be highly influenced by availability of parental support (Barber, Stolz, Olsen, \& Maughan, 2005) and behavioral and psychological control by the parents (Pettit \& Laird 2002; Suldo \& Huebner, 2004). In the field of parental behavioral control, certain views suggest a need to identify the multi-dimensional nature of parental behavioral control (Smetana \& Daddis, 2002) and to discriminate various aspects of parental behavioral control. Shek (2006) argued that it is also important to examine whether adolescent children are satisfied with the parental behavioral control imposed on them. The adolescent children's satisfaction is important because it determines the extent to which they are willing to be socialized (Darling \& Steinberg, 1993), which is a factor mediating the effect of parenting on adolescent psychological well-being. Most of the studies in this area were conducted predominately in Western contexts with few studies conducted in different Chinese communities (Shek, 2006). 
With specific reference to the domain of parent-adolescent relational qualities, cross-sectional research findings suggest that at least three areas of parent-child relational qualities might influence child's satisfaction with parental control (Shek, 2005, 2006). The first area is whether the adolescent child trusts the parent. The reasoning is that if the child trusts the parent, he or she will be more satisfied with the parental behavioral control. The second area is whether the child has a perception that the parent trusts him/her. It can be conjectured that if the child does not feel trusted, parental behavioral control would be seen negatively.

Unfortunately, a survey of the literature shows that the role of trust has been inadequately covered. For example, Noack, Kerr, and Olah (1999) believed that trust has received little empirical attention, despite the apparent importance of socialization of trust. According to the theoretical propositions of the attachment theories (e.g., Bailham \& Harper, 2004; Bowlby, 1988) and attachment-based family therapies (Diamond \& Stern, 2003), it was expected that a higher level of child's trust (which symbolizes a higher sense of security) would add to better parent-child relational qualities (e.g., satisfaction with parental behavioural control). Various studies show that mutual trust between parents and adolescents is significantly related to parentchild relational qualities (Shek, 2006).

Besides mutual trust between parents and their adolescent children, readiness of the child to communicate with the parents (e.g., the child's voluntary disclosure) also influences his or her satisfaction with parental behavioural control. It can be logical that as the child's readiness to communicate with the parents increases, parental knowledge would also increase. In addition, the child's readiness to communicate with the parents would facilitate the socialization process in which parents would find it easier to control the behaviour of the child. Empirically, several studies have noted that the child's readiness to communicate with the parents (i.e., child's voluntary disclosure) was positively related to parental knowledge as well as parental control (Crouter \& Head, 2002; Kerr \& Stattin, 2000).

Various studies indicated a relation between parental control and symptoms of anxiety and depression among adolescents (Barber \& Harmon, 2002; Wijsbroek, Hale III, Raaijmakers \& Meeus, 2011). But satisfaction with parental control has not been given much emphasis with regard to mental health problems among adolescents. Although satisfaction with parental control has been investigated in Chinese culture (Shek, 2005), It was hardly studied in India. Therefore, it was considered one of the important variables to be taken to find out its relationship with mental health of adolescents.

The rapidly changing social, political and economical scenario in the world has not left Indian family untouched. It is going through structural and functional modifications that have a bearing on adolescent's socialization and parent- child relations. Weakening of social support from kinship, movement of women empowerment, exposure to media, increasing competitive 
demands of the market economy and higher standards of achievement are a few aspects that have changed the family dynamics in the recent past. Although earlier studies have discovered the relationship between parental behaviours and adolescents' mental health, there is strong need to check the connection between satisfaction with parental control and adolescents' mental health during current cultural globalization era.

The current study is aimed to examine and compare the satisfaction with parental control (paternal and maternal) and mental health problems (e.g., anxiety, depression) among adolescent boys and girls. Study also aimed to elucidate the relationship of satisfaction with parental control along with anxiety and depression among adolescents. An attempt has also been made to understand the contribution of satisfaction with parental control (paternal and maternal) in the prediction of adolescents' mental health problems (anxiety and depression). In summary three research questions are included in the study.

1. Are there gender differences regarding satisfaction with parental control (paternal and maternal) and mental health problems (anxiety and depression)?

2. Is there a relationship between adolescents' satisfaction with parental control and their mental health problems?

3. To what extent satisfaction with parental control is predicting anxiety and depression among adolescent boys and girls.

On the basis of literature it was expected that,

1. Boys and girls would significantly differ with regard to satisfaction with parental control (paternal and maternal) and various mental health problems (anxiety and depression).

2. Ssatisfactions with parental control (paternal and maternal) would be negatively associated with anxiety and depression among adolescent boys and girls.

\section{METHODOLOGY}

\section{Sample}

The present study followed a sample of 400 adolescents with age range of 15 to 19 years. Sample included equal number of boys $(\mathrm{N}=200$; Mean age=16.01) and girls $(\mathrm{N}=200$; Mean age=16.06) studying in different government and private high school and intermediate colleges of Varanasi city, U.P., India. Adolescents belonging to middle socioeconomic status were selected in the sample on the basis of scores of Socio-Economic Status Scale (Dubey \& Nigam, 2005). Adolescents from both nuclear and joint family structure were integrated in the sample. It was the demand of the study to have data about both mother and father; therefore, adolescents having both parents alive and staying together were selected.

In addition to a demographic data schedule (age, gender, family structure and size of the family, parents’ education and parents’ occupation etc.) the following measures were used. 


\section{Measures}

Satisfaction with Parental Control Scale developed by Shek (2005), was used to assess satisfaction with parental control. Paternal Control Scale included four items to assess the satisfaction with paternal control $(\alpha=.78)$. Identical items with reference to "my mother" are used to assess the respondent's satisfaction with maternal control that formed the Satisfaction with Maternal Control Scale $(\alpha=.75)$. The items in the scale are rated on a 4-point Likert-type scale ranging from 1 (strongly disagree) to 4 (strongly agree).

General Health Questionnaire (GHQ) constructed by Goldberg and Hollies (1979), was used to assess two dimensions of mental health problems, viz. anxiety and depression. It consists of 14 items. This is a four-point rating scale. Each item was scored from 1 to 4. The response alternatives have been arranged in such a way that higher scores on each dimension indicate greater health problems. Item-total correlation of each subscale ranges from 0.40 to 0.85 and the Alpha -coefficient ranges from 0.75 to 0.84 .

\section{Procedure}

Aforesaid questionnaires were administered to adolescents individually. Instructions were clearly explained to each participant before the actual administration of the scales and their queries (if any) were attended properly. Participants were allowed to take their own time to respond all the questionnaires. In addition to it each participant was requested to ensure that they have responded to each and every item of the scales. After collection of questionnaires from the participants scoring have been done according to instructed in the respective manual or planned in the course of the development.

\section{RESULTS}

In the first step of the analysis, mean, standard deviations and t-values were computed to find out the differences between boys and girls on all the measured variables under study. Correlation coefficients have been computed to examine the extent of relationship between satisfaction with parental control (paternal and maternal) and mental health problems (anxiety and depression) separately for boys and girls. Further, to examine the role of satisfaction with paternal and maternal control, multiple regression analysis (step wise) was performed with the predictor (satisfaction with paternal and maternal control) and criterion variables (anxiety and depression).

Results in terms of mean, SD and t-value for satisfaction with parental control (paternal and maternal) and mental health problems (anxiety and depression) among adolescents boys and girls were presented in table-1. 
Mental Health Problems among Adolescents in Relationship to Satisfaction with Parental Control

Table 1: Mean, SD, and t- Value for Satisfaction with Paternal Control (Paternal and Maternal) and Various Mental Health Problems (Anxiety and Depression) of Boys and Girls

\begin{tabular}{|c|c|c|c|}
\hline & Boys (N=200) & Girls (N=200) & \multirow{2}{*}{$\mathbf{t}$} \\
\cline { 2 - 3 } & Mean (SD) & Mean (SD) & \multirow{2}{*}{1.251} \\
\hline $\begin{array}{c}\text { Satisfaction with Paternal } \\
\text { Control }\end{array}$ & $12.98(2.83)$ & $12.62(2.92)$ & .179 \\
\hline $\begin{array}{c}\text { Satisfaction with Maternal } \\
\text { Control }\end{array}$ & $12.72(2.82)$ & $12.77(2.76)$ & .557 \\
\hline Anxiety & $11.85(4.30)$ & $12.09(4.14)$ & .047 \\
\hline Depression & $11.13(4.32)$ & $11.11(4.12)$ & \\
\hline
\end{tabular}

Perusal of table 1 revealed that mean score of boys for satisfaction with paternal control was observed to be higher than girls, while mean score for satisfaction with maternal control of girls was higher than boys. Boys and girls did not differ significantly on perception of satisfaction with paternal and maternal control. Table-1 also represents the mean, SD, and t-value for anxiety and depression among adolescents boys and girls. Examination of table-1 revealed that girls in comparison to boys had higher mean scores for anxiety, whereas boys in comparison to girls obtained higher mean scores for depression. There were no significant difference between boys and girls for anxiety and depression. Therefore, the first hypothesis which assumed that boys and girls would significantly differ with regard to satisfaction with parental control (paternal and maternal) and various mental health problems (anxiety and depression) wasn't supported by the findings.

The results regarding the association of satisfaction with paternal and maternal control along with anxiety and depression were summarized in table-2.

Table 2: Correlation of Satisfaction with Paternal/Maternal Control Scores with Anxiety and Depression of Boys $(N=200)$ and Girls $(N=200)$

\begin{tabular}{|c|c|c|c|}
\hline & & Anxiety & Depression \\
\hline \multirow{2}{*}{$\begin{array}{l}\text { Satisfaction with Paternal } \\
\text { Control }\end{array}$} & Boys & $-.178^{*}$ & $-.177^{*}$ \\
\hline & Girls & $-.226^{* *}$ & $-.209^{* *}$ \\
\hline \multirow{2}{*}{$\begin{array}{l}\text { Satisfaction with Maternal } \\
\text { Control }\end{array}$} & Boys & $-.165^{*}$ & $-.169^{*}$ \\
\hline & Girls & $-.301^{* *}$ & $-.284^{* *}$ \\
\hline
\end{tabular}

${ }^{*} \mathrm{p}<0.05 \quad * * \mathrm{p}<0.01$

The results presented in table 2 indicated that satisfaction with paternal control has significant negative association with anxiety and depression of boys and girls. Satisfaction with maternal control has also significant negative relation with anxiety and depression of boys and girls. Thus, overall pattern of correlation coefficients supported the hypothesis, which assumed that satisfaction with parental control would be negatively related with anxiety and depression of adolescents.

(c) The International Journal of Indian Psychology, ISSN 2348-5396 (e)| ISSN: 2349-3429 (p) | 158 
Multiple regression analysis (step wise): The Role of Satisfaction with Parental Control (Paternal and Maternal) in Predicting Anxiety and Depression among Adolescent Boys and Girls.

The results of multiple regression analysis (step wise) regarding the role of satisfaction with parental control in explaining variance in anxiety and depression of boys and girls were presented together in table 3.

Table 3: Results of Step-Wise Multiple Regression Analysis Using Satisfaction with Parental Control (Paternal and Maternal) as Predictor and anxiety and depression as Criterion

\begin{tabular}{|c|c|c|c|c|c|c|c|}
\hline \multicolumn{8}{|c|}{ 1.Criterion variable: Anxiety } \\
\hline Model & Variable & $\mathbf{R}$ & $\mathbf{R}^{2}$ & $\mathbf{R}^{2}$ adj. & $\mathbf{R}^{2}$ change & Beta & F Ratio \\
\hline 1. & $\begin{array}{l}\text { Satisfaction with } \\
\text { maternal control }\end{array}$ & .231 & .053 & .051 & .053 & $-.231^{* * *}$ & $22.41 * * *$ \\
\hline 2 & $\begin{array}{l}\text { Satisfaction with } \\
\text { paternal control }\end{array}$ & .251 & .063 & .058 & .010 & $-.115^{*}$ & $13.37 * * *$ \\
\hline \multicolumn{8}{|c|}{ 2. Criterion variable: Depression } \\
\hline Model & Variable & $\mathbf{R}$ & $\mathbf{R}^{2}$ & $\mathbf{R}^{2}$ adj. & $\mathbf{R}^{2}$ change & Beta & F Ratio \\
\hline 1 & $\begin{array}{l}\text { Satisfaction with } \\
\text { maternal control }\end{array}$ & .225 & .051 & .048 & .051 & $-.225 * * *$ & $21.17 * * *$ \\
\hline
\end{tabular}

An examination of the table 3 reveals that satisfaction with maternal control emerged as the more significant predictor of anxiety and depression. It is also evident from the table that the beta coefficients for the significant predictors were negative. Satisfaction with maternal control explained $5.3 \%$ of the total variance in anxiety scores and $5.1 \%$ of the total variance in depression. Satisfaction with paternal control explained $1.0 \%$ of the total variance in anxiety scores. Satisfaction with paternal control didn't emerge as significant predictor of depression of adolescents. Thus, findings of regression analysis suggested that satisfaction with maternal control emerged as a more significant predictor of adolescents' anxiety and depression than satisfaction with paternal control.

\section{DISCUSSION}

Obtained data was analyzed to meet the objectives formulated for the study. First, the analysis of gender differences, second, the analysis of relationships between variables and finally, the analysis was performed to evaluate the role of relative significance of predictor (satisfaction with paternal control satisfaction with maternal control) in explaining variances in anxiety and depression. Reviewed literature indicated that effect of satisfaction of parental control was rarely explored in Indian research. The majority of studies that have examined the impact of satisfaction with parental control have been conducted in Chinese cultures. Most of the conclusions in previous studies about the association between parenting style and adolescents' mental health are based on parent or observer reports of parenting. Adolescents' perception is

(C) The International Journal of Indian Psychology, ISSN 2348-5396 (e)| ISSN: 2349-3429 (p) | 159 
considered more important for adolescents' wellbeing rather than actual parental behaviours. Thus, this study is based on adolescents' reports about their satisfaction with parental control and mental health status.

The first objective of the study was to examine and compare the satisfaction with parental control (paternal and maternal) and mental health problems (e.g., anxiety, depression) among adolescent boys and girls. Results revealed that boys and girls were more or less equal with regard satisfaction with parental control (paternal and maternal) and anxiety and depression. Gender was found to be non-significant indicating that the pattern of satisfaction with parental control (paternal and maternal) and mental health problems are same for adolescents' boys and girls. Gender differences regarding satisfaction with parental control (paternal and maternal) indicated that in these days Indian parents doesn't treat their sons and daughter differently. Indian adolescents are satisfied with parental control as they give respect to elders and comply with them (Jain \& Bhatt, 2004). Previous studies also support the present findings regarding insignificant gender difference for anxiety and depression (Ghaderi, Kumar \& Kumar, 2009).

The second objective of the study was to examine the relationship of satisfaction with parental control along with anxiety and depression among adolescent boys and girls. The overall pattern of correlation coefficients revealed that satisfaction with parental control has significant negative association with anxiety and depression of adolescents. It indicated that adolescents who are more satisfied with their parental control exhibit better mental health status. The adolescent's satisfaction is important because it determines the extent to which they are willing to be socialized, which is a factor mediating the effect of parenting on adolescent developmental outcomes (Shek, 2005). We may explain our result in light of Attachment theory, which suggests that children who experience positive relationships with their parents are more securely attached to those parents; are more likely to be securely attached in later relationships; and are at lower risk of psychological health problems (Bowlby, 1988). These findings extend support to the previous observations which show that positive parent-child relationship promotes positive wellbeing and behaviors of adolescents (Richards \& Hatch, 2011; Stafford, Kuh, Gale, Mishra \& Richards, 2016).

With specific reference to the domain of parent-adolescent relational qualities, studies suggest that at least three areas of parent-child relational qualities might influence child's satisfaction with parental control. The first area is whether the adolescent child trusts the parent. It implies that if the child trusts the parent, he/she will be more satisfied with the parental control. The second area is whether the child has a perception that the parent trusts him/her. It can be inferred that if the child does not feel trusted, parental control would be seen negatively. Empirically, few studies show that mutual trust between parents and adolescents is significantly related to parentchild relational qualities. Readiness of the child to communicate with the parents (e.g., the child's 
voluntary disclosure) also influences his/her satisfaction with parental behavioural control (Shek, 2006).

The third objective of the present study was to examine the role of satisfaction with parental control in predicting anxiety and depression of adolescent boys and girls. Multiple regression analysis (step wise) revealed that satisfaction with maternal control emerged as significant predictor of both anxiety and depression among adolescents. Satisfaction with paternal control also predicted significant variance in depression scores of adolescents. Analyses of multiple regression implies that satisfaction with parental control, mainly of satisfaction with maternal control have significant contribution in explaining variance in anxiety and depression of adolescents. Attachment theories (e.g., Bailham \& Harper, 2004; Bowlby, 1988) support that child trust and sense of security is more related with mothers' parenting. Cross cultural studies also suggested that maternal control gives feelings of security and acceptance to Indian adolescents (Albert, Trommsdorff \& Mishra, 2004). Thus, adolescents feel satisfied with maternal control as well as with themselves. Results indicated that if adolescents trust on parents and feel that parent control their behaviour for betterment, they feel satisfied with parental control and possess better mental health.

Study gives more importance on satisfaction with maternal control. Results can be explained in context of Indian culture where mothers are often physically and verbally affectionate, more nurturing and spending more time with children at home; fathers tend to express caring indirectly (Jambunathan \& Counselman, 2002; Rudy \& Grusec, 2006). Satisfaction with maternal control gives a sense of caring and acceptance to adolescents, which positively influence the mental health of adolescents. Satisfaction with paternal control was also found important for adolescents' mental health. Contemporary research suggested that middle-class fathers in urban areas of India are increasingly becoming more nurturing, affectionate, and interactive in the daily lives of their young children, suggesting a cultural shift in parenting approaches for fathers (Roopnarine, Talukder, Jain, Joshi, \& Srivastav, 1990). Today fathers generally in the urban contexts of India expect an ideal father to be aware of and address their children's needs, and to be a friend, teacher, and guide to their children (Saraff \& Srivastava, 2008).

Although parental behaviour has been largely investigated, this study makes an important contribution by exploring the association between satisfaction with parental control and mental health problems. Study revealed that adolescents having satisfaction with parental control exhibited less anxiety and depression. Findings regarding importance of satisfaction with parental control exhibited for adolescents' mental health may be useful for clinicians and counsellors dealing with adolescents' psychological problems. Current findings have important implications for parent education programs, counselling and family intervention. 


\section{Acknowledgments}

The author appreciates all those who participated in the study and helped to facilitate the research process.

Conflict of Interests: The author declared no conflict of interests.

\section{REFERENCES}

Albert, I., Trommsdorff, G. \& Mishra, R. (2004). Parenting and Adolescent Attachment in India and Germany. In Zheng, G., Leung, K. and Adair, J. G.,M Perspectives and progress in contemporary cross-cultural psychology: 17th International Congress of the International Association for Cross-cultural Psychology: 97-108.

Bailham, D. \& Harper, P. B. (2004). Attachment theory and mental health. London, United Kingdom: Jessica Kingsley Publishers.

Barber, B. K. \& Harmon, E. L. (2002). Violating the self: Parental psychological control of children and adolescents. In B. K. Barber (Ed.), Intrusive parenting: How psychological control affects children and adolescents (pp. 15-52). Washington, DC: American Psychological Association.

Barber, B. K., Stolz, H. E., Olsen, J. A. \& Maughan, S. L. (2005). Parental support, psychological control, and behavioral control: Assessing relevance across time, method, and culture. Monographs of the Society for Research in Child Development, 70, 1-151.

Baumrind, D. \& Black, A. E. (1967). Socialization practices associated with dimensions of competence in preschool boys and girls. Child Development, 38, 291-327.

Bowlby, J. (1973). Attachment and loss (Vol. 2). New York: Basic Books.

Bowlby, J. (1988). Developmental psychiatry comes of age. American Journal of Psychiatry, $145,1-10$.

Chorpita, B. F. \& Barlow, D. H. (1998). The development of anxiety: The role of control in the early environment. Psychological Bulletin, 124, 3-21.

Clarke, G. N., DeBar, L. L., \& Lewinsohn, P. M. (2003). Cognitive-behavioral group treatment for adolescent depression. In A. E. Kazdin \& J.R. Weisz (Eds.), Evidence-based psychotherapies for children and adolescents (pp. 120-134). New York, NY, US: Guilford Press.

Crouter, A. C. \& Head, M. (2002). Parental monitoring and knowledge of children. In Bornstein, M. H. (Ed.) Handbook of Parenting: Vol.3: Being and Becoming a parent (2nd ed.), 461483, Lawrence Erlbaum.

Darling, N. \& Steinberg, L. (1993). Parenting style as context: An integrative model. Psychological Bulletin, 113, 487-496.

Diamond, G. S. \& Stern, R. (2003). Attachment based family therapy: Repairing attachment ruptures. In S. Johnson \& V. E. Whiffen (eds.), Attachment Process in Couple and Family Therapy. Guilford Press: New York.

Dubey, L. N. \& Nigam, B. (2005). Socio-Economic Status Scale (Urban). Arohi Manovigyan Kendra, Datta \& Chaddha Enclav, South Civil Lines, Jabalpur.

Essau, C.A., Conradt, J. \& Petermann, F. (2000). Frequency, comorbidity, and psychosocial impairment of depressive disorders in adolescents. Journal of Adolescent Research, 15, 470-481. 
Flouri, E. \& Buchanan, A. (2003). The role of father involvement in children's later mental health. Journal of Adolescence, 26(1), 63-78.

Ghaderi, A. R., Kumar, V., G., \& Kumar, S. (2009). Depression, Anxiety and Stress among the Indian and Iranian Students. Journal of the Indian Academy of Applied Psychology, 5, 3337.

Goldberg, D. \& Hollies, V. F. (1979). A scaled version of the general health questionnaire. Psychological Medicine, 9, 139-145.

Grotevant, H. D. \& Cooper, C. R. (1986). Individuation in family relationships: a perspective on individual differences in the development of identity and role-taking skill. Human Development, 29, 82-100.

Heaven, P. C. L., Newbury, K. \& Mak, A. (2004). The impact of adolescent and parental characteristics on adolescents levels of delinquency and depression. Personality and Individual Differences, 36, 173-185.

Jain, P. C. \& Bhatt, M. (2004).Consumer behaviour in Indian context. S. Chand, New Delhi.

Jambunathan, S. \& Counselman, K. (2002). Parenting attitudes of Asian Indian mothers living in the United States and in India. Early Child Development \& Care, 172, 657-662.

Kerr, M. \& Stattin, H. (2000). What parents know, how they know it, and several forms of adolescent adjustment: Further support for a reinterpretation of monitoring. Developmental Psychology, 36, 366-380.

McLeod, B. D., Wood, J. J. \& Weisz, J. R. (2007). Examining the association between parenting and childhood anxiety: A meta-analysis. Clinical Psychology Review, 27, 155-172.

Noack, P., Kerr, M. \& Olah, A. (1999). Family relations in adolescence. Journal of Adolescence, 22, 713-717.

Pavlidis, K. \& McCauley, E. (2001). Autonomy and relatedness in family interactions with depressed adolescents. Journal of Abnormal Child Psychology, 29, 11-21.

Pettit, G.S. \& Laird, R.D. (2002). Psychological control and monitoring in early adolescence: The role of parental involvement and prior child adjustment. In B. K. Barber (Ed.), Intrusive parenting: How psychological control affects children and adolescents (pp. 97123). Washington, DC: American Psychological Association.

Pillai, A., Patel, V., Cardozo, P., Goodman, R., Weiss, H. A. \& Andrew, G. (2008). Nontraditional lifestyles and prevalence of mental disorders in adolescents in Goa, India. The British Journal of Psychiatry, 192, 45-51.

Rapee, R. M. (2001). The development of generalised anxiety. In M. W. Vasey, M. R. Dadds (Eds), The developmental psychopathology of anxiety (pp. 481-503). New York: Oxford University Press.

Richards M. \& Huppert F. A. (2011). Do positive children become positive adults? Evidence from a longitudinal birth cohort study. The Journal of Positive Psychology, 75-87.

Roopnarine, J. \& Suppal, P. (2003). Kakar's psychoanalytic interpretation of childhood: The need to emphasize the father and multiple caregivers in the socialization equation. In D. Sharma (Ed.), Childhood, family and socio-cultural change in India: Reinterpreting the inner world (pp. 115-137) New Delhi: Oxford.

Rudy, D. \& Grusec, J. E. (2006). Authoritarian parenting in individualist and collectivist groups: Associations with maternal emotion and cognition and children's self-esteem. Journal of Family Psychology 20, 68-78.

Saraff, A. \& Srivastava, H.C. (2008). Envisioning fatherhood: Indian fathers' perception of an ideal father. Population Review, 47, 45-59. 
Shek, D. T. L. (2005). Perceived parental control processes, parent child relational qualities, and psychological well-being in Chinese adolescents with and without economic disadvantage. The Journal of Genetic Psychology, 166, 171-188.

Shek, D. T. L. (2006). Perceived parental behavioral control and psychological control in Chinese adolescents in Hong Kong. American Journal of family Therapy, 34, 163-176.

Smetana, J. G. \& Daddis, C. (2002). Domain specific antecedent of parental psychological control and monitoring: The role of parenting beliefs and practices. Child Development, 73, 563-580.

Srinath, S, Girimaji, S. C., Gururaj, G., Seshadri, S, Subbakrishna, D. K., Bhola P, \& Kumar, N. (2005). Epidemiological study of child and adolescent psychiatric disorders in urban and rural areas of Bangalore, India. Indian Journal of Medicine, 122, 67 -79.

Stafford, M., Kuh, D. L., Gale, C.R., Mishra, G. \& Richards, M. (2016). Parent-child relationships and offspring's positive mental wellbeing from adolescence to early older age. Journal of Positive Psychology, 11(3), 326-337.

Suldo, S. M. \& Huebner, E. S. (2004). The role of life satisfaction in the relationship between authoritative parenting dimensions and adolescent problem behavior. Social Indicators Research, 66, 165 - 195.

Wijsbroek, S. A., Hale III, W.W., Raaijmakers, Q. A. W. \& Meeus, W. H. J. (2011). The direction of effects between perceived parental behavioural control and psychological control and adolescents' self-reported GAD and SAD symptoms. European Child Adolescent Psychology, 20, 361-371.

Wilkinson, R. B. (2004). The role of parental and peer attachment in the psychological health and self-esteem of adolescents. Journal of Youth and Adolescence, 33, 479-493

Wood, J. J. (2006). Parental intrusiveness and children's separation anxiety in a clinical sample. Child Psychiatry \& Human Development, 37, 73-87.

Wood, J. J., McLeod, B. D., Sigman, M., Hwang, W. C. \& Chu, B. C. (2003). Parenting and childhood depression: Theory, empirical findings, and future directions. Journal of Child Psychology and Psychiatry, 44, 134-151.

How to cite this article: Tiwari V, Verma S (2017), Mental Health Problems among Adolescents in Relationship to Satisfaction with Parental Control, International Journal of Indian Psychology, Volume 4, Issue 2, No. 88, ISSN:2348-5396 (e), ISSN:2349-3429 (p), DIP:18.01.076/20170402, ISBN:978-1-365-74161-6 